Use and effect of paediatric life support skills for paediatric arrest

EDITOR,- The article on use and effect of paediatric life support skills for paediatric arrest in the accident and emergency ( $A \& E$ ) department has three conclusions ${ }^{1}$ : (1) the poor outcome, well supported in the literature ${ }^{23} ;(2)$ advanced life support training in the receiving hospital has not improved the outcome; and (3) the timing of initiation of life support is a critical factor affecting outcome.

A large recent study of children presenting with apnoea with or without a cardiac output found that good prognostic indicators in cluded a short time between arrest and arrival at hospital, a cardiac output at presentation and a short duration of resuscitation in the A\&E department (two or fewer doses of adrenaline or resuscitated within 20 minutes). ${ }^{2}$

Training of pre-hospital personnel in life support thus would seem indicated, but this must highlight the importance of not unduly delaying transportation by untimely out of hospital interventions.

Owing to the poor outcome of paediatric cardiopulmonary arrests, courses such as the Advanced Paediatric Life Support (APLS) and the Paediatric Advanced Life Support (PALS) have been developed, focusing on structured intervention in critically ill children with emphasis on early recognition and aggressive management to prevent cardiac arrest. The teaching, however, is aimed at hospital personnel so the Advanced Life Support Group (ALSG) have now also developed the Pre-hospital Paediatric Life Support (PHPLS) course, specifically designed to allow the continuity of care from the prehospital arena to the $A \& E$ department. It uses most of the core material of APLS but incorporates specific pre-hospital problems with emphasis on prompt transportation to hospital. The standard is comparable to APLS and it is suitable for any doctors, paramedics, and nurses working with critically ill children outside hospital. Further information can be obtained from Sue Wieteska, National Coordinator, ALSG, Second floor, The Dock Office, Salford Quays, Manchester M5 2XB.

IAN MACONOCHIE St Mary's Hospital, Praed Street, London W2 $1 \mathrm{NY}$ FIONA JEWKES

Cardiff Royal Infirmary Newport Road, Cardiff CF2 $1 S Z$

1 Hassan TB. Use and effect of paediatric advanced life support skills for paediatric arrest in the A\&E department. J Accid Emerg Med the A\&E depar

2 Schindler MB, Bohn D, Cox P, et al. Outcome of out-of-hospital cardiac or respiratory arrest in out-of-hospital cardiac or respiratory arrest

3 O'Rourke P. Outcome of children who are apnoeic and pulseless in the emergency room Crit Care Med 1986;14:466-8.

\section{Accidental digital injection of adrenaline from an autoinjector device}

EDITOR,-We managed a 39 year old day care assistant recently with accidental digital injection of adrenaline from an autoinjector device. He had opened the device mistaking it for a pen. He came to us with the device still impaled in his right index finger

From our experience with this case, we find that it is better to use saline or a clear antiseptic solution to prepare the skin than use Beta- dine. The latter stains the skin and interferes with the observation of reversal of skin pallor seen within a few minutes after phentolamine injection. ${ }^{12}$

We gave a digital block with $1 \%$ lignocaine to extract the needle of the device from the distal phalanx of his right index finger. We then infiltrated his finger pulp space (at the site of impalement) with phentolamine $1.5 \mathrm{mg}$ (in increments of $0.5 \mathrm{mg}$ ) diluted in $1 \%$ lignocaine. ${ }^{1-3}$ The anaesthetic effect of the digital block was persistent after the vascularity was restored and interfered with the study of return of sensation. ${ }^{2}$ In retrospect we wonder whether local infiltration of phentolamine with lignocaine by itself could have provided sufficient analgesia to remove the impaled needle and treat the injury as well. ${ }^{12}$

It is interesting to note that many victims of this accidental injury have been paramedica personnel, law enforcers, and carers who have failed to recognise this device. ${ }^{3}$ This device is marketed as EpiPen (standard and junior versions). The label on the device and the accompanying literature describes how it has to be used and it is usually dispensed with dummy trainer. However the device looks like and can easily be mistaken for a marker pen Accidental digital injection is an avoidable injury with grievous consequence, which could possibly be prevented by appropriate modification of the external appearance/nam of the autoinjector device so as to caution the unwary.

KALYAN S MURALI NADEEM NAYEEM Accident and Emergency Department University Hospital, Lewisham, London SE13 6LH

1 McGovern SJ. Treatment of accidental digital injection of adrenaline from an auto-injector device. J Accid Emerg Med 1997;14:379-80.

2 Markovchick V, Burkhart KK. The reversal of the ischaemic effects of epinephrine on the finger with local injections of phentolamine. Emerg Med 1991;9:323-4.

3 Hardy SJ, Agostini DE. Accidental epinephrin auto-injector induced digital ischemia reversed by phentolamine digital block. J Am Osteopath Assoc 1995;95:377-8.

EDITOR,-We read with interest the suggested treatment of the accidental injection of adrenaline from an autoinjector device, with local injection of phentolamine. ${ }^{1}$ We agree this is a growing problem in the UK as the device is now widely prescribed. It was suggested the reversal of the adrenaline by the application of topical glyceryl trinitrate is ineffective. To the contrary, we have treated successfully two cases of such injury to the thumb with glyceryl trinitrate. A 19 year old women was treated with glyceryl trinitrate patches (Nitodur, Schering-Plough Ltd, $10 \mathrm{mg}$ patch) over 24 hours. The digit changed from white to pink and became warm in one hour. The patch was applied for 48 hours. A 27 year old women was treated with glyceryl trinitrate spray (Nitro Lingual Pump Spray, Lipha, $400 \mu$ glyceryl trinitrate in ethanol). After a gauze soaked in glyceryl trinitrate was applied to the digit, the capillary refill became normal in one hour. Although phentolamine is stocked in the majority of the hospital pharmacies, is no readily available in the accident and emergency department. The reversal of adrenaline induced ischaemic injuries by glyceryl trinitrate in our two cases suggests this simple treatment should still be considered initially. If successful, this avoids the use of phentolamine, an unfamiliar drug, with potential risks.

G LEE

P C THOMAS

Accident and Emergency Department fohn Radcliffe Hospital, Oxford OX3 9DU

\section{The author replies}

I very much welcome this letter by Lee and Thomas and the finding that colleagues are indeed experiencing an increasing number of patients presenting with accidental digital injection of adrenaline from autoinjector devices. I read with interest that they have been able to successfully reverse adrenaline induced digital ischaemia with a glyceryl trinitrate patch and swab soaked with spray. Glyceryl trinitrate is believed to exert its vasodilator effect through nitric oxide stimulating an increase in cyclic guanosine monophosphate, which in turn induces smooth muscle relaxation by lowering the free calcium concentration in the cytosol. In its action on vascular muscle venous dilation predominates over dilation of the arterioles. ${ }^{2}$ Given that adrenaline causes vasoconstriction via $\alpha$ receptors on arterioles, a non-selective $\alpha$ blocker would appear a more pharmacologically sound treatment option. This does not exclude glyceryl trinitrate causing vasodilation via another pathway. It is recognised that the therapeutic effect of glyceryl trinitrate topically occurs between 30-60 minutes and this would fit with their finding of vasodilation taking place within one hour. ${ }^{3}$ I feel however that in the cases they describe it is probable that the accidental injection took place away from the digital bundle and therefore the adrenaline had a weaker constrictive effect, which was successfully reversed by the glyceryl trinitrate.

This problem is likely to present more frequently to accident and emergency departments and there is a need for a treatment protocol, which was one of the conclusions of my paper. ${ }^{1}$ Unfortunately as with many aspects of emergency medicine it does not lend itself easily to a randomised control trial being performed. From what Lee and Thomas report it may be worth trying glyceryl trinitrate if the patient presents shortly after accidental injection ( $<3$ hours), the injection is unlikely to be intra-arterial or phentolamine is not immediately available, however if there was no therapeutic effect within 60 minutes of application of glyceryl trinitrate phentolamine must be used without delay.

1 McGovern SJ. Treatment of accidental digita injection of adrenaline device. J Accid Emerg Med 1997;14:379-80.

2 Thadami U, Whitsett T. Relationship of pharma cokinetic and pharmacodynamic properties of the organic nitrates. Clin Pharmacokinet 1988, 15:32-43.

3 Bogaert MG. Clinical pharmacokinetics of glyceryl trinitrate following the use of systemic and topical preparations. Clin Pharmacokinet 1987, $12: 1-11$

\section{Nutmeg intoxication}

EDITOR,--In these days of increasingly sophisticated designer drugs, policed with "zero tolerance", we wish to report a case of recreational drug ingestion involving a substance freely available in every supermarket-the spice nutmeg. 